Research Article

\title{
The Decomposition Theorem for a Fuzzy Quasinorm
}

\author{
Rui Gao $\mathbb{D}$, Xinxin $\mathrm{Li}(\mathbb{D}$, and Jianrong $W u(\mathbb{D}$ \\ College of Mathematics Science, Suzhou University of Science and Technology, Suzhou, Jiangsu 215009, China \\ Correspondence should be addressed to Jianrong Wu; jrwu@mail.usts.edu.cn
}

Received 1 September 2020; Revised 27 September 2020; Accepted 17 October 2020; Published 3 November 2020

Academic Editor: Nan-Jing Huang

Copyright (C) 2020 Rui Gao et al. This is an open access article distributed under the Creative Commons Attribution License, which permits unrestricted use, distribution, and reproduction in any medium, provided the original work is properly cited.

This paper deals with fuzzy quasinormed spaces in the sense of Alegre and Romaguera. After introducing the concept of the family of star quasiseminorms, we prove the decomposition theorem for a fuzzy quasinorm with general $t$-norm, characterize fuzzy quasinorms in terms of families of star quasiseminorms, and establish the connection between the fuzzy quasinorm and the family of quasinorms.

\section{Introduction}

In 1984, Katsaras [1] first introduced an idea of fuzzy norm on a linear space. In 1992, Felbin [2] introduced the concept of fuzzy norm on a linear space whose associated metric is Kaleva and Seikkala type [3]. Inspired by the notion of probabilistic metric spaces, Kramosil and Michalek [4], in 1975, introduced the notion of fuzzy metric, a fuzzy set in the Cartesian product $X \times X \times(-\infty, \infty)$ satisfying certain conditions. In 1994, Cheng and Mordeson [5] introduced an idea of fuzzy norm on a linear space in such a manner that the corresponding fuzzy metric is of Kramosil and Michalek type. Following Cheng and Mordeson, in 2003, Bag and Samanta [6] introduced a definition of fuzzy norm and proved the decomposition theorem of fuzzy norm to a family of crisp norms. This concept has been used in developing fuzzy functional analysis and its applications. Bag and Samanta [7] first considered a general t-norm in the definition of fuzzy normed linear space which was introduced in [6] and proved that if $t$-norm is chosen other than "min," then the decomposition theorem of fuzzy norm may not hold. Since the decomposition theorem plays an important role in developing fuzzy functional analysis, it is worthy to establish a new kind of decomposition theorem of fuzzy norm with general t-norm. That is one of the goals of this article.

On the other hand, Bag and Samanta [6] stated that given an ascent family of norms on a real linear space $X$, a fuzzy norm can be determined on $X$. Furthermore, in 2009, Sadeqi and Kia [8] proved that a separating family of seminorms introduces a fuzzy norm in general, but it is not true in classical analysis. In 2014, Alegre and Romaguera [9] also dealt with fuzzy normed spaces in the sense of Cheng and Mordeson and characterized fuzzy norms in terms of ascending and separating families of seminorms. It is worth noting that the fuzzy norms mentioned above require a strong restriction on the particular choice of "min" for $t$-norm. Therefore, a natural query arises: how far the results of fuzzy normed linear spaces can be established with the fuzzy norm in its general form, i.e., waiving the restricted " $m i n$ " $t$-norm in the triangle inequality. To deal with this problem is another goal of this article.

With the exception of symmetry of a fuzzy norm in [6], Alegre and Romaguera [10] introduced the concept of fuzzy quasinorm. They proved some results, such as the uniform boundedness theorem, in fuzzy quasinormed spaces in [11].

In this paper, we are going to conduct research in the framework of fuzzy quasinormed linear space introduced in [10]. After investigating some properties of " $\alpha$-quasiseminorms" corresponding to a fuzzy quasinorm, we introduce a concept of a family of star quasiseminorms in Section 3. The novelty of this definition is the validity of a decomposition theorem for a fuzzy quasinorm $N$ with general $t$-norm into the associated family of star quasiseminorms, denoted by $P_{N}$. Additionally, we prove that an increasing and separating family of star quasiseminorms $P$ induces a fuzzy quasinorm, 
denoted by $N_{P}$. Moreover, we show that a family of star quasiseminorms coincides with the family of star quasiseminorms associated to the fuzzy quasinorm induced by $P$ (see Theorem 4); meanwhile, a fuzzy quasinorm $N$ coincides with the fuzzy quasinorm induced by the family of star quasiseminorms associated to $N$ (see Theorem 5). That is, $P=P_{N_{P}}$ and $N=N_{P_{N}}$. Combining Theorems 4 and 5, we get the decomposition theorem for a fuzzy quasinorm with general t-norm, characterize fuzzy quasinorms in terms of families of star quasiseminorms, and establish the connection between the fuzzy quasinorm and the family of quasinorms.

\section{Preliminaries}

Throughout this paper, the symbols $\wedge$ and $\vee$ mean the operator "min" and "max," respectively. Let $Z^{+}$be the set of all positive integers, $R=(-\infty, \infty), R^{+}=(0, \infty)$. [12].

First, let us recall the concept of continuous $t$-norms

Definition 1. A binary operation $*:[0,1] \times[0,1] \longrightarrow[0,1]$ is a continuous t-norm if it satisfies the following conditions:

(T1): * is associative and commutative

(T2): * is continuous

(T3): $a * 1=a, \forall a \in[0,1]$

(T4): $a * b \leq c * d$ whenever $a \leq c$ and $b \leq d$ and $a, b, c, d \in[0,1]$

The following are examples of some t-norms that are frequently used as fuzzy intersections defined for all $a, b \in[0,1]$.

(i) Standard intersection: $a \Delta_{1} b=a \wedge b$

(ii) Algebraic product: $a \Delta_{2} b=a b$

(iii) Bounded difference: $a \Delta_{3} b=\max \{a+b-1,0\}$

Definition 2 (see [10]). A fuzzy quasinorm on a real linear space $X$ is a pair $(N, *)$, or $N$ for simplicity, such that $*$ is a continuous $t$-norm and $N$ is a fuzzy set in $X \times[0, \infty)$ satisfying the following conditions for every $x, y \in X$ :

(i) (FQN1): $N(x, 0)=0$

(ii) (FQN2): $N(x, t)=N(-x, t)=1$ for all $t>0$ if and only if $x=0$

(iii) (FQN3): $N(c x, t)=N(x, t / c)$ for all $c \in R^{+}$

(iv) (FQN4): $N(x, t) * N(y, s) \leq N(x+y, t+s)$ for all $s, t \geq 0$

(v) (FQN5): $\quad N(x, \cdot): \quad[0, \infty) \longrightarrow[0,1]$ is left continuous

(vi) (FQN6): $\lim _{t \rightarrow \infty} N(x, t)=1$

Remark 1. We point out that (FQN2) and (FQN4) imply that $N(x, \cdot)$ is increasing.

The following condition will be used in the paper:
(FQN7): for $x \neq 0, N(x, \cdot)$ is strictly increasing on $\{t: 0<N(x, t)<1\}$.

A fuzzy norm [1] on a real linear space $X$ is a fuzzy quasinorm $(N, *)$ on $X$ such that $N(c x, t)=N(x, t /|c|)$ for all $x \in X$ and $c \in R /\{0\}$. Recall that if in Definition 2, we put $*=\wedge$, then one has the notion of a fuzzy norm as given by Cheng and Morderson [5].

By a fuzzy (quasi-) normed space, we mean a triple $(X, N, *)$ such that $X$ is a real linear space and $(N, *)$ is a fuzzy (quasi-) norm on $X$.

Each fuzzy quasinorm $(N, *)$ on $X$ defines a topology (denoted by $\tau_{N}$ ) on $X$ by taking all open balls as a base:

$$
\left\{B_{N}(x, r, t): x \in X, r \in(0,1), t>0\right\},
$$

where $B_{N}(x, r, t)=\{y \in X: N(y-x, t)>1-r\} . B_{N}(x, r, t)$ is said to be an open ball with center $x$ and radius $r$. It is easy to see that the topology $\tau_{N}$ is $T_{0}$.

In the rest of the paper, the notation $\alpha_{n} \uparrow \alpha$ ( $\alpha_{n} \downarrow \alpha$, resp.) means that an increasing (decreasing, resp.) sequence of real numbers $\left\{\alpha_{n}\right\}$ convergences to a real number $\alpha$.

\section{Quasiseminorm Structures in a Fuzzy Quasinormed Space}

It is well known that a quasinorm on a real linear space $X$ is a function $p: X \longrightarrow[0, \infty)$ satisfying the conditions: for all $x, y \in X$ and $c \in R^{+}$,

(i) (QN1): $p(x)=p(-x)=0 \Rightarrow x=0$

(ii) (QN2): $p(c x)=c p(x)$

(iii) (QN3): $p(x+y) \leq p(x)+p(y)$

If $p$ satisfies only the conditions (QN2) and (QN3), then it is called a quasiseminorm.

Remark 2. From (QN2), we get $p(0)=0$.

Proposition 1. Let $(X, N, *)$ be a fuzzy quasinormed space, and let $\alpha \in(0,1)$. The function $\|\cdot\|_{\alpha}: X \longrightarrow[0, \infty)$ is given by

$$
\|x\|_{\alpha}=\inf \{t>0: N(x, t) \geq \alpha\}
$$

Then, for all $x \in X$ and $t \in R^{+}$,

(1) $\|x\|_{\alpha}$ is increasing with respect to $\alpha \in(0,1)$

(2) $\|x\|_{\alpha}=\sup \{t>0: N(x, t)<\alpha\}$

(3) $N(x, t) \geq \alpha$ implies that $\|x\|_{\alpha} \leq t$, and equivalently, $\|x\|_{\alpha}>t$ implies that $N(x, t)<\alpha$

(4) $N(x, t)>\alpha$ implies that $\|x\|_{\alpha}<t$, and $\|x\|_{\alpha}<t$ implies that $N(x, t) \geq \alpha$

The proof is direct and omitted. $\left\{\|\cdot\|_{\alpha}: \alpha \in(0,1)\right\}$ is called the family of " $\alpha$-quasiseminorms" corresponding to fuzzy quasinorm $N$ and denoted by $P_{N}$.

Proposition 2. Let $(X, N, *)$ be a fuzzy quasinormed space, and let $x \in X$. If $N(x, \cdot)$ is continuous and satisfies (FQN7), then $\|x\|_{\alpha}$ is strictly increasing with respect to $\alpha \in(0,1)$. 
Proof. Let $\alpha_{1}, \alpha_{2} \in(0,1)$ with $\alpha_{1}<\alpha_{2}$. Since $N(x, \cdot)$ is strictly increasing and continuous, there exist $t_{1}, t_{2} \in R^{+}$ such that $N\left(x, t_{1}\right)=\alpha_{1}, \quad N\left(x, t_{2}\right)=\alpha_{2} \quad$ and $N(x, t) \in\left(\alpha_{1}, \alpha_{2}\right)$ when $t \in\left(t_{1}, t_{2}\right)$. It is easy to see that $\|x\|_{\alpha_{2}}=t_{2}>t_{1}=\|x\|_{\alpha_{1}}$. Thus, $\|x\|_{\alpha}$ is strictly increasing with respect to $\alpha \in(0,1)$.

Proposition 3. Let $(X, N, *)$ be a fuzzy quasinormed space, and let $x \in X$. Then, the following assertions are equivalent:

(1) $N(x, \cdot)$ satisfies (FQN7)

(2) $\|x\|_{\alpha}=\inf \{t>0: N(x, t)>\alpha\}=\sup \{t>0: N(x, t) \leq \alpha\}$ for each $\alpha \in(0,1)$

(3) $N(x, t)>\alpha$ if and only if $\|x\|_{\alpha}<t$, that is, $N(x, t) \leq \alpha$ if and only if $\|x\|_{\alpha} \geq t$

Proof

(i) (1) $\Rightarrow$ (3): from Proposition 1 (4), we only have to show $\|x\|_{\alpha}<t$ implies $N(x, t)>\alpha$. In fact, if $\|x\|_{\alpha}<t$, by the definition of $\|x\|_{\alpha}$, there is $0<t_{1}<t$ such that $N\left(x, t_{1}\right) \geq \alpha$. Thus $N(x, t)>\alpha$ whenever $N(x, \cdot)$ satisfies (FQN7).

(ii) $(3) \Rightarrow(2)$ : let

$$
t_{0}=\inf \{t>0: N(x, t)>\alpha\} .
$$

In the light of (2), we get $\|x\|_{\alpha} \leq t_{0}$. If $\|x\|_{\alpha}<t_{0}$, we have $N\left(x, t_{0}\right)>\alpha$ from (3). Since $N(x, \cdot)$ is left continuous, there is $\delta>0$ such that $N\left(x, t_{0}-\delta\right)>\alpha$, which conflicts with the definition of $t_{0}$. Thus,

$$
\begin{aligned}
\|x\|_{\alpha} & =t_{0}=\inf \{t>0: N(x, t)>\alpha\} \\
\inf \{t>0: N(x, t)>\alpha\} & =\sup \{t>0: N(x, t) \leq \alpha\} .
\end{aligned}
$$

can be shown by using the similar technique used in Proposition 1 (2).

(iii) $(2) \Rightarrow(1)$ : suppose that $N(x, \cdot)$ is not strictly increasing. Then, there exist $t_{1}, t_{2} \in\{t: 0<N(x, t)<1\}$ such that $t_{1}<t_{2}$ and $N(x, \cdot) \equiv \alpha_{0}$ on $\left[t_{1}, t_{2}\right]$. Thus,

$\sup \left\{t>0: N(x, t) \leq \alpha_{0}\right\} \geq t_{2}>t_{1} \geq \inf \left\{t>0: N(x, t) \geq \alpha_{0}\right\}$

$=\|x\|_{\alpha_{0}}$,

which conflicts with the supposition (2).

Definition 3. Let $I \subseteq R$. A family of real-valued maps $\left\{d_{i}: i \in I\right\}$ will be called

(1) lower semicontinuous (shortly LSC) if for any $i \in I$, $d_{i}=\inf _{j>i} d_{j}$

(2) upper semicontinuous (shortly USC) if for any $i \in I$, $d_{i}=\sup _{j<i} d_{j}$
(3) continuous if it is both LSC and USC

The following lemma is obvious.

Lemma 1. Let I be an interval in $R$. An increasing family of real-valued maps $\left\{d_{i}: i \in I\right\}$ is

(1) LSC if and only for any sequence $\left\{\alpha_{n}\right\}$ in I with $\alpha_{n} \downarrow \alpha \in \in I, d_{\alpha}=\lim _{n \longrightarrow \infty} d_{\alpha_{n}}$

(2) USC if and only for any sequence $\left\{\alpha_{n}\right\}$ in I with $\alpha_{n} \uparrow \alpha \in \in I, d_{\alpha}=\lim _{n \longrightarrow \infty} d_{\alpha_{n}}$

Proposition 4. Let $(X, N, *)$ be a fuzzy quasinormed space, and let $\alpha \in(0,1), t>0 .\|x\|_{\alpha}$ is defined by (2). Then,

(1) $\left\{\|\cdot\|_{\alpha}: \alpha \in(0,1)\right\}$ is USC

(2) $\left\{\|\cdot\|_{\alpha}: \alpha \in(0,1)\right\}$ is LSC if and only if $N(x, \cdot)$ satisfies (FQN7)

Proof

(1) Let $\alpha_{n}, \alpha \in(0,1)$ and $\alpha_{n} \uparrow \alpha$. From Proposition 1 (1), $\left\{\|x\|_{\alpha_{n}}\right\}$ is increasing and $\lim _{n \longrightarrow \infty}\|x\|_{\alpha_{n}} \leq\|x\|_{\alpha}$. If $\lim _{n \longrightarrow \infty}\|x\|_{\alpha_{n}}<\|x\|_{\alpha}$, then there is a $t_{0}>0$ such that $\lim _{n \longrightarrow \infty}\|x\|_{\alpha_{n}}<t_{0}<\|x\|_{\alpha}$, and hence, $\|x\|_{\alpha_{n}}<t_{0}$ for all $n \in Z^{+}$, which together with Proposition 1 (4) follows that $N\left(x, t_{0}\right) \geq \alpha_{n}$ for all $n \in Z^{+}$. Thus, $N\left(x, t_{0}\right) \geq \alpha$. Hence, $\|x\|_{\alpha} \leq t_{0}$. This is a contradiction. Thus, $\lim _{n \rightarrow \infty}\|x\|_{\alpha_{n}}=\|x\|_{\alpha}$.

(2) Suppose $N(x, \cdot)$ satisfies (FQN7). Let $\alpha_{n}, \alpha \in(0,1)$ and $\alpha_{n} \uparrow \alpha$; then, $\lim _{n \longrightarrow \infty}\|x\|_{\alpha_{n}} \geq\|x\|_{\alpha}$. Suppose $\lim _{n \longrightarrow \infty}\|x\|_{\alpha_{n}}>\|x\|_{\alpha}$. Then, there is a $t_{0}>0$ such that $\lim _{n \longrightarrow \infty}\|x\|_{\alpha_{n}}>t_{0}>\|x\|_{\alpha}$, and then, $\|x\|_{\alpha_{n}}>t_{0}$ for all $n \in Z^{+}$. By Proposition 1 (3), we have $N\left(x, t_{0}\right)<\alpha_{n}$ for all $n \in Z^{+}$. Therefore, $N\left(x, t_{0}\right) \leq \alpha$.

Case 1: $N\left(x, t_{0}\right)<\alpha$. From Proposition 1 (2), we get $\|x\|_{\alpha}>t_{0}$. This is a contradiction.

Case 2: $N\left(x, t_{0}\right)=\alpha$. Since $N(x, \cdot)$ satisfies (FQN7), $N\left(x, t_{0}-\varepsilon\right)<\alpha$ for any $\varepsilon>0$. Then, $\|x\|_{\alpha}>t_{0}-\varepsilon$ from Proposition 1 (3). Hence, $\|x\|_{\alpha} \geq t_{0}$. This is a contradiction.

Combining the abovementioned discussion, we know $\lim _{n \longrightarrow \infty}\|x\|_{\alpha_{n}}=\|x\|_{\alpha}$. From Lemma 1, we know that $\left\{\|\cdot\|_{\alpha}: \alpha \in(0,1)\right\}$ is LSC.

Now, we suppose $\left\{\|\cdot\|_{\alpha}: \alpha \in(0,1)\right\}$ is LSC. Let $t_{1}, t_{2} \in R^{+}$with $t_{1}<t_{2}$. Then, $N\left(x, t_{1}\right) \leq N\left(x, t_{2}\right)$. Suppose that $N\left(x, t_{1}\right)=N\left(x, t_{2}\right)$; then, $N(x, \cdot) \equiv \alpha_{0}$ on $\left[t_{1}, t_{2}\right]$. Let $m=\sup \left\{t: N(x, t)=\alpha_{0}\right\}$; then,

$$
\|x\|_{\alpha_{0}}=\inf \left\{t>0: N(x, t) \geq \alpha_{0}\right\} \leq t_{1}<t_{2} \leq m .
$$

Since $N(x, \cdot)$ is left continuous, $N(x, m)=\alpha_{0}$. For any $\alpha^{\prime}>\alpha_{0}$ and any $t>0$ with $N(x, t) \geq \alpha^{\prime}$, we have $t>m$; hence, $\|x\|_{\alpha^{\prime}}=\inf \left\{t>0: N(x, t) \geq \alpha^{\prime}\right\} \geq m$. Let $\left\{\alpha_{n}\right\} \subseteq(0,1)$ be a strictly decreasing sequence with $\alpha_{n} \downarrow \alpha_{0}$; then, $\lim _{n \longrightarrow \infty}\|x\|_{\alpha_{n}} \geq m>\|x\|_{\alpha_{0}}$, which conflicts with the supposition. Thus, $N\left(x, t_{1}\right)<N\left(x, t_{2}\right)$. That is, $N(x, \cdot)$ satisfies (FQN7). 
Definition 4. Let $X$ be a linear space and $*$ be a continuous $t$-norm. For each $\alpha \in(0,1), p_{\alpha}$ is a function from $X$ to $R^{+}$. $P=\left\{p_{\alpha}: \alpha \in(0,1)\right\}$ is called a family of star quasiseminorms if it satisfies the conditions: for all $x, y \in X, \alpha, \beta \in(0,1)$, and $c \in[0, \infty)$,

$$
\begin{aligned}
& (* \mathrm{QN} 1): p_{\alpha}(c x)=c p_{\alpha}(x) \\
& (* \mathrm{QN} 2): p_{\alpha * \beta}(x+y) \leq p_{\alpha}(x)+p_{\beta}(y)
\end{aligned}
$$

If $P$ satisfies the condition $(* \mathrm{QN} 3): p_{\alpha}(x)=0$ for every $\alpha \in(0,1)$ implies $x=0$, then $P$ is said to be separating.

Remark 3. From ( $* \mathrm{QN} 1)$, we know $p_{\alpha}(0)=0$ for every $\alpha \in(0,1)$.

Remark 4. If $*=\wedge$, then a family of star quasiseminorms is just a family of quasiseminorms.

The following result is obvious:

Proposition 5. Let $P=\left\{p_{\alpha}: \alpha \in(0,1)\right\}$ be a family of star quasiseminorms. For each $x \in X$, let

$$
\begin{aligned}
U_{P}(x)= & \left\{U_{P}\left(x ; \alpha_{1}, \alpha_{2}, \ldots, \alpha_{n} ; \varepsilon\right):\right. \\
& \left.\cdot \varepsilon>0 ; \alpha_{1}, \alpha_{2}, \ldots, \alpha_{n} \in(0,1), \quad n \in Z^{+}\right\},
\end{aligned}
$$

where

$$
\begin{aligned}
U_{P}\left(x ; \alpha_{1}, \alpha_{2}, \ldots, \alpha_{n} ; \varepsilon\right)= & \left\{y \in X: p_{\alpha_{i}}(y-x)<\varepsilon, \alpha_{i} \in(0,1),\right. \\
& i=1,2, \ldots, n\} \\
\cap_{i=1}^{n}\left\{y \in X: p_{\alpha_{i}}(y-x)<\varepsilon, \alpha_{i} \in(0,1)\right\} & \\
& .\left\{y \in X: p_{\max \left\{\alpha_{i}: 1 \leq i \leq n\right\}}(y-x)<\varepsilon\right\} .
\end{aligned}
$$

Then, $U_{P}(x)$ is a basis of neighborhoods of $x$.

The topology taking $U_{P}(x)$ as a basis of neighborhoods of $x$ is said to be the topology induced by $P$ and denoted by $\tau_{P}$. It is easy to show that $\tau_{P}$ is $T_{0}$ if $P$ is separating.

Theorem 1. Let $(X, N, *)$ be a fuzzy quasinormed space. $P_{N}=\left\{\|\cdot\|_{\alpha}: \alpha \in(0,1)\right\}$ where $\|\cdot\|_{\alpha}$ is defined by (2) for all $\alpha \in(0,1)$. Then,

(1) $P_{N}$ is a separating family of $*$ quasiseminorms

(2) the topology $\tau_{P_{N}}$ induced by $P_{N}$ coincides the topology $\tau_{N}$

\section{Proof}

(1) Let $x, y \in X, \alpha \in(0,1)$, and $c \in R^{+}$.

$(* \mathrm{QN} 1):\|c x\|_{\alpha}=\inf \{t>0: N(c x, t) \geq \alpha\}=\inf \{t>$ $0: N(x, t / c) \geq \alpha\}=\inf \left\{c t^{\prime}>0: N\left(x, t^{\prime}\right) \geq \alpha\right\}$ $=c\|x\|_{\alpha}$.

(*QN2): for any $t_{1}>\|x\|_{\alpha}$ and $t_{2}>\|y\|_{\beta}$, from the definition of $\|\cdot\|_{\alpha}$, there exist $t_{1}^{*}, t_{2}^{*}>0$ such that $t_{1}^{*}<t_{1}, \quad t_{2}^{*}<t_{2}, \quad N\left(x, t_{1}^{*}\right) \geq \alpha \quad$ and $\quad N\left(y, t_{2}^{*}\right) \geq \beta$. Hence,

$$
N\left(x+y, t_{1}^{*}+t_{2}^{*}\right) \geq N\left(x, t_{1}^{*}\right) * N\left(y, t_{2}^{*}\right) \geq \alpha * \beta .
$$

Therefore,

$$
\|x+y\|_{\alpha * \beta}=\inf \{t>0: N(x+y, t) \geq \alpha * \beta\} \leq t_{1}^{*}+t_{2}^{*}<t_{1}+t_{2} .
$$

By the arbitrariness of $t_{1}$ and $t_{2}$, we know that $\|x+y\|_{\alpha * \beta} \leq\|x\|_{\alpha}+\|y\|_{\beta}$.

(*QN3): if $\|x\|_{\alpha}=0$ for every $\alpha \in(0,1)$, then $\|x\|_{\alpha}<t$ for all $t>0$; hence, $N(x, t) \geq \alpha$ from Proposition 1 (4). By the arbitrariness of $\alpha \in(0,1)$, we get $N(x, t)=1$ for all $t>0$. In light of (FQN2), we have $x=0$.

(2) For any $x, y \in X, \quad r \in(0,1)$ and $t>0$, if $N(y-x, t)>1-r$, then $\|y-x\|_{1-r}<t$ from Proposition 1 (4). Therefore, $B_{N}(x, r, t) \subseteq U_{P_{N}}(x ; 1-r ; t)$.

On the other hand, for any $x, y \in X, \alpha \in(0,1)$, and $t>0$, if $\|y-x\|_{\alpha}<t$, then there is $0<t_{1}<t$ such that $N\left(y-x, t_{1}\right) \geq \alpha$ from (2). Hence,

$$
N(y-x, t) \geq N\left(y-x, t_{1}\right)>1-r \text {, }
$$

for any $1>r>1-\alpha$. So, $U_{P_{N}}(x ; \alpha ; t) \subseteq B_{N}(x, r, t)$. Combining the abovementioned discussion, we get $\tau_{N}=\tau_{P_{N}}$.

Remark 5. $P_{N}=\left\{\|\cdot\|_{\alpha}: \alpha \in(0,1)\right\}$ is said to be the family of star quasiseminorms associated with the fuzzy quasinorm $(N, *)$.

Example 1. Let $(X,\|\cdot\|)$ be a quasinormed space, and let $N$ : $X \times[0, \infty) \longrightarrow[0,1]$ given by

$$
N(x, t)= \begin{cases}0, & t=0, \\ \frac{t}{t+\|x\|}, & t>0,\end{cases}
$$

for all $x \in X$. It is well known that $(N, *)$ is a fuzzy quasinorm on $X$, where $*$ is any continuous t-norm. This fuzzy quasinorm is called the standard fuzzy quasinorm induced by $\|\cdot\|$. Obviously, $N(x, t) \geq \alpha$ is equivalent to $t \geq(\alpha / 1-\alpha)\|x\|$ for any $\alpha \in(0,1)$. So, it follows from (2) that

$$
\|x\|_{\alpha}=\frac{\alpha}{1-\alpha}\|x\| .
$$

Therefore, $P_{N}=\{\alpha / 1-\alpha\|\cdot\|: \alpha \in(0,1)\}$.

Theorem 2. Let $(X, N, *)$ be a fuzzy quasinormed space. If for any $x \in X$, there exists $t>0$ such that $N(x, t)=1$. The function $\|\cdot\|_{1}: X \longrightarrow[0, \infty)$ is given by

$$
\|x\|_{1}=\inf \{t>0: N(x, t)=1\},
$$

then, $\|\cdot\|_{1}$ is a quasinorm on $X$. Moreover, 


$$
\|x\|_{1}=\sup _{\alpha \in(0,1)}\|x\|_{\alpha}=\lim _{\alpha \longrightarrow 1-0}\|x\|_{\alpha} .
$$

Proof

(i) (QN1): suppose $\|x\|_{1}=\|-x\|_{1}=0$. From (2), for any $t>0$, there exists $0<t^{\prime}, t^{\prime \prime}<t$ such that $N\left(x, t^{\prime}\right)=1$ and $\quad N\left(-x, t^{\prime \prime}\right)=1 ; \quad$ therefore, $\quad N(x, t)$ $=N(-x, t)=1$, which together with (FQN2) implies that $x=0$.

(ii) (QN2): let $\lambda>0$. Then,

$$
\begin{aligned}
\|\lambda x\|_{1} & =\inf \{t>0: N(\lambda x, t)=1\} \\
& =\inf \left\{t>0: N\left(\frac{x, t}{\lambda}\right)=1\right\} \\
& =\inf \left\{\lambda t^{\prime}>0: N\left(x, t^{\prime}\right)=1\right\}=\lambda\|x\|_{1} .
\end{aligned}
$$

(iii) (QN3): take any $t_{1}>\|x\|_{1}$ and $t_{2}>\|y\|_{1}$. From (2), there exist $t_{1}^{*}, t_{2}^{*}>0$ such that $t_{1}^{*}<t_{1}, t_{2}^{*}<t_{2}$, $N\left(x, t_{1}^{*}\right)=1$ and $N\left(y, t_{2}^{*}\right)=1$. Thus,

$$
N\left(x+y, t_{1}^{*}+t_{2}^{*}\right) \geq N\left(x, t_{1}^{*}\right) * N\left(y, t_{2}^{*}\right)=1 * 1=1 .
$$

Therefore,

$$
\|x+y\|_{1}=\inf \{t>0: N(x+y, t)=1\} \leq t_{1}^{*}+t_{2}^{*} .
$$

By the arbitrariness of $t_{1}$ and $t_{2}$, we know that $\|x+y\|_{1} \leq\|x\|_{1}+\|y\|_{1}$. Thus, $\|\cdot\|_{1}$ is a quasinorm.

Now, we prove (15). Since $\left\{\|x\|_{\alpha}: \alpha \in(0,1)\right\}$ is increasing, it is easy to see that

$$
\|x\|_{1} \geq \sup _{\alpha \in(0,1)}\|x\|_{\alpha}=\lim _{\alpha \longrightarrow 1-0}\|x\|_{\alpha}
$$

for all $x \in X$. For any $x \in X$, from the definition of $\|x\|_{1}$, we know $t>\|x\|_{1}-\varepsilon$ when $N(x, t)=1$. Thus, $N(x, \cdot)<1$ on $\left(0,\|x\|_{1}-\varepsilon\right]$. Hence, $N\left(x,\|x\|_{1}-\varepsilon\right)<1$. Take $\alpha_{0}>0$ such that $N\left(x,\|x\|_{1}-\varepsilon\right)<1-\alpha_{0}$. By Proposition 1 (4), we get $\|x\|_{1-\alpha_{0}} \geq\|x\|_{1}-\varepsilon$. Consequently,

$$
\|x\|_{1} \leq \sup _{\alpha \in(0,1)}\|x\|_{\alpha} .
$$

The inequalities (19) and (20) imply equation (15).

Theorem 3. Let $P=\left\{\|\cdot\|_{\alpha}: \alpha \in(0,1)\right\}$ be an increasing separating USC family of star quasiseminorms on a real linear space $X$. For all $x \in X$ and $t>0$, let $X \times[0, \infty) \longrightarrow[0,1]$ be given by

$$
N_{P}(x, t)= \begin{cases}0, & t=0, \\ \sup \left\{\alpha \in(0,1):\|x\|_{\alpha}<t\right\}, & t>0 .\end{cases}
$$

Then,

(1) the pair $\left(N_{P}, *\right)$ is a fuzzy quasinorm on $X$
(2) the topology $\tau_{N_{P}}$ induced by fuzzy quasinorm $N_{P}$ coincides the topology $\tau_{P}$ induced by $P$

Proof

(1) (FQN1) is obvious.

(FQN2): if $N_{p}(x, t)=1$ for all $t>0$, then $\|x\|_{\alpha}<t$ for all $\alpha \in(0,1)$ from (21). Therefore, $\|x\|_{\alpha}=0$ for all $\alpha \in(0,1)$. Since $P$ is separating, $x=0$. Conversely, if $x=0$, then $\|x\|_{\alpha}=0<t$ for all $t>0$ from Remark 3 . Hence, $N_{P}(x, t)=N_{P}(-x, t)=1$ from (21).

(FQN3): let $c \in R^{+}$. From $(* \mathrm{QN} 1)$, we have

$$
\begin{aligned}
N_{P}(c x, t) & =\sup \left\{\alpha \in(0,1):\|c x\|_{\alpha}<t\right\} \\
& =\sup \left\{\alpha \in(0,1):\|x\|_{\alpha}<\frac{t}{c}\right\} \\
& =N_{P}(x, t / c) .
\end{aligned}
$$

(FQN4):let $x, y \in X$ and $s, t>0$. Set $N_{P}(x, t)=\beta$, $N_{P}(y, s)=\gamma$. For any $\varepsilon>0$ with $\varepsilon<\min \{\beta, \gamma\}$, there exist $\alpha^{\prime}, \alpha^{\prime \prime} \in(0,1)$ such that $\alpha^{\prime}>\beta-\varepsilon, \alpha^{\prime \prime}>\gamma-\varepsilon$, $\|x\|_{\alpha^{\prime}}<t$, and $\|y\|_{\alpha^{\prime \prime}}<s$. Therefore, $\|x\|_{\beta-\varepsilon}<t$ and $\|y\|_{\gamma-\varepsilon}<s$. Hence,

$$
\|x+y\|_{(\beta-\varepsilon) *(\gamma-\varepsilon)} \leq\|x\|_{\beta-\varepsilon}+\|y\|_{\gamma-\varepsilon}<t+s .
$$

It follows from (21) that

$$
N_{P}(x+y, t+s) \geq(\beta-\varepsilon) *(\gamma-\varepsilon) .
$$

By the arbitrariness of $\varepsilon>0$ and the continuity of $*$, we know that

$N_{P}(x+y, t+s) \geq \beta * \gamma=N_{P}(x, t) * N_{P}(y, s)$.

(FQN5): it is easy to see that $N_{P}(0, \cdot) \equiv 1$, and hence, it is continuous. Now, take $x_{0} \in X /\{0\}$ and $t_{0}>0$ arbitrarily. If $N_{P}\left(x, t_{0}\right)=0$, then $N_{P}(x, t)=N_{P}\left(x, t_{0}\right)=0$ for all $t<t_{0}$. So, $N_{P}(x, \cdot)$ is left continuous at $t_{0}$. On the other hand, if $0<N_{P}\left(x, t_{0}\right) \leq 1$. Given $\varepsilon>0$ arbitrarily, from (21), there exists $\alpha_{0} \in(0,1)$ such that $\|x\|_{\alpha_{0}}<t_{0}$ and $N_{P}\left(x, t_{0}\right)-\varepsilon<\alpha_{0}$. For any $t$ with $\|x\|_{\alpha_{0}}<t<t_{0}$, we have $N_{P}(x, t) \geq \alpha_{0}$ by (21). Hence, $N_{P}\left(x, t_{0}\right)-N_{P}(x, t) \leq N_{P}\left(x, t_{0}\right)-\alpha_{0}<\varepsilon$. Therefore, $N_{P}(x, \cdot)$ is left continuous at $t_{0}$.

(FQN6): let $x \in X$ and let $\varepsilon>0$. There exists $\alpha_{0} \in(0,1)$ such that $1-\alpha_{0}<\varepsilon$. For any $t>\|x\|_{\alpha_{0}}$, we have $\quad N_{p}(x, t) \geq \alpha_{0}>1-\varepsilon$. Therefore, $\lim _{t \rightarrow \infty} N_{P}(x, t)=1$.

(2) For all $x, y \in X, \quad r \in(0,1)$, and $t>0$, if $N_{P}(y-x, t)>1-r$, then there is $\alpha \in(1-r, 1)$ such that $\|y-x\|_{\alpha}<t$; therefore, $B_{N_{P}}(x, r, t) \subseteq U_{P}(x ; \alpha ; t)$.

On the other hand, for all $x, y \in X, \alpha \in(0,1)$, and $t>0$, if $\|y-x\|_{\alpha}<t$, then $N_{P}(y-x, t) \geq \alpha$ from the definition of 
$N_{P}$. Hence, $N_{P}(y-x, t)>1-r$ for any $1>r>1-\alpha$. Therefore, $U_{P}(x ; \alpha ; t) \subseteq B_{N_{P}}(x, r, t)$.

Combining the abovementioned discussion, we get $\tau_{N_{P}}=\tau_{P}$.

Remark 6 . The abovementioned fuzzy quasinorm $\left(N_{P}, *\right)$ is said to be induced by the family of star quasiseminorms $P$.

Theorem 4. Let $P=\left\{\|\cdot\|_{\alpha}: \alpha \in(0,1)\right\},\left(N_{P}, *\right)$ be as in Theorem 3.3. $P_{N_{P}}=\left\{\langle\cdot\rangle_{\alpha}: \alpha \in(0,1)\right\}$ is the family of star quasiseminorms associated with $\left(N_{P}, *\right)$. Then, $\langle x\rangle_{\alpha}=\|x\|_{\alpha}$ for all $x \in X$ and $\alpha \in(0,1)$. That is, $P_{N_{P}}=P$.

Proof. For any $t>\|x\|_{\alpha}$, we know $N_{P}(x, t) \geq \alpha$ from (21), which together with Proposition 1 (3) implies that $\langle x\rangle_{\alpha} \leq t$. By the arbitrariness of $t$, we have $\langle x\rangle_{\alpha} \leq\|x\|_{\alpha}$.

For any $t>\langle x\rangle_{\alpha}$, from (2), we know there exists $t_{0} \in(0, t)$ such that $N_{P}\left(x, t_{0}\right) \geq \alpha$.

(i) Case 1: $N_{P}\left(x, t_{0}\right)>\alpha$. From (21), there exists $\alpha_{0} \in(\alpha, 1) \quad$ such that $\|x\|_{\alpha_{0}}<t_{0}$. Since $\left\{\|x\|_{\alpha}: \alpha \in(0,1)\right\} \quad$ is increasing, we have $\|x\|_{\alpha}<\|x\|_{\alpha_{0}}<t_{0}<t$. By the arbitrariness of $t$, we have $\|x\|_{\alpha} \leq\langle x\rangle_{\alpha}$

(ii) Case 2: $N_{P}\left(x, t_{0}\right)=\alpha$. From (21), there exists a strictly increasing sequence $\left\{\alpha_{n}\right\} \subseteq(0, \alpha)$ with $\alpha_{n} \uparrow \alpha$ such that $\|x\|_{\alpha_{n}}<t_{0}$. Noting that $P=\left\{\|\cdot\|_{\alpha}: \alpha \in(0,1)\right\}$ is USC, we have $\|x\|_{\alpha} \leq t_{0}<t$. By the arbitrariness of $t$, we have $\|x\|_{\alpha} \leq\langle x\rangle_{\alpha}$.

Combining the abovementioned discussion, we get $\|x\|_{\alpha}=\langle x\rangle_{\alpha}$.

Theorem 5. Let $(X, N, *)$ be a fuzzy quasinormed space. Then, $N_{P_{N}}=N$.

Proof. Let $P_{N}=\left\{\|\cdot\|_{\alpha}: \alpha \in(0,1)\right\}$ be the family of star quasiseminorms induced by the fuzzy quasinorm $N$, and let $x \in X$.

It is obvious that $N_{P_{N}}(x, 0)=0=N(x, 0)$.

Let $t>0$. From (21) and Proposition 1 (4), we obtain that $N_{P_{N}}(x, t) \leq N(x, t)$ directly. Now, we are going to show that $N_{P_{N}}(x, t) \geq N(x, t)$. Without loss of generality, we suppose that $N(x, t)>0$. We take a strictly increasing sequence $\left\{\alpha_{n}\right\} \subseteq(0, N(x, t))$ with $\alpha_{n} \uparrow N(x, t)$. From Proposition 1 (4), we get $\|x\|_{\alpha_{n}}<t$. So, $\quad N_{P_{N}}(x, t) \geq \alpha_{n}$. Therefore, $N_{P_{N}}(x, t) \geq N(x, t)$.

Combining the abovementioned discussion, we get $N_{P_{N}}(x, t)=N(x, t)$ for all $x \in X$ and $t \geq 0$, and hence, $N_{P_{N}}=N$.

\section{Conclusions}

This paper introduces a concept of the family of star quasiseminorms. With this new concept, the decomposition theorem for a fuzzy quasinorm with general $t$-norm is established, and the quasiseminorm structures in a fuzzy quasinormed space are revealed. Based on these results, the connection between the fuzzy quasinorm and the quasinorm is established. The proposed method provides a powerful tool to study the fuzzy functional analysis. Many results about the fuzzy functional analysis may be obtained easily from the corresponding versions in the functional analysis with the help of the decomposition theorem for a fuzzy quasinorm. Conversely, some topics about the functional analysis can be investigated in the view of the fuzzy functional analysis. For example, the characterizations of those pseudotopological linear spaces [13] that are fuzzy quasinormable may be investigated deeply. Moreover, the decomposition technique proposed in this paper may be applied in other research fields such as fuzzy Lie algebras (see [14-16]) and Pythagorean fuzzy set theory [17].

\section{Data Availability}

No data were used to support this study.

\section{Conflicts of Interest}

The authors declare that they have no conflicts of interest.

\section{Authors' Contributions}

Jianrong $\mathrm{Wu}$ was responsible for conceptualization, methodology, funding acquisition, and review and editing. Rui Gao and Xinxin Li contributed to formal analysis, investigation, and original draft preparation. All authors have read and agreed to the published version of the manuscript.

\section{Acknowledgments}

The authors acknowledge the support of the National Natural Science Foundation of China under Grant no. 11371013. The authors are grateful to the referees for their valuable comments which led to the improvement of this paper.

\section{References}

[1] A. K. Katsaras, "Fuzzy topological vector spaces II," Fuzzy Sets and Systems, vol. 12, no. 2, pp. 143-154, 1984.

[2] C. Felbin, "Finite dimensional fuzzy normed linear space," Fuzzy Sets and Systems, vol. 48, no. 2, pp. 239-248, 1992.

[3] O. Kaleva and S. Seikkala, "On fuzzy metric spaces," Fuzzy Sets and Systems, vol. 12, no. 3, pp. 215-229, 1984.

[4] I. Kramosil and J. Michalek, "Fuzzy metrics and statistical metric spaces," Kybernetika, vol. 11, pp. 336-344, 1975.

[5] S. C. Cheng and J. N. Mordeson, "Fuzzy linear operators and fuzzy normed linear spaces," Bulletin of the Calcutta Mathematical Society, vol. 86, no. 5, pp. 429-436, 1994.

[6] T. Bag and S. K. Samanta, "Finite dimensional fuzzy normed linear spaces," Journal of Fuzzy Mathematics, vol. 11, pp. 687-705, 2003.

[7] T. Bag and S. K. Samanta, "Finite dimensional fuzzy normed linear spaces," Annals of Fuzzy Mathematics and Informatics, vol. 6, no. 2, pp. 271-283, 2013.

[8] I. Sadeqi and F. S. Kia, "Fuzzy normed linear space and its topological structure," Chaos, Solitons \& Fractals, vol. 40, pp. 2576-2589, 2009.

[9] C. Alegre and S. Romaguera, "The Hahn-Banach extension theorem for fuzzy normed spaces revisited," Abstract and Applied Analysis, vol. 2014, Article ID 151472, 7 pages, 2014. 
[10] C. Alegre and S. Romaguera, "Characterizations of metrizable topological vector spaces and their asymmetric generalizations in terms of fuzzy (quasi-)norms," Fuzzy Sets and Systems, vol. 161, no. 16, pp. 2181-2192, 2010.

[11] C. Alegre and S. Romaguera, "On the uniform boundedness theorem in fuzzy quasi-normed spaces," Fuzzy Sets and Systems, vol. 282, pp. 143-153, 2016.

[12] B. Schweizer and A. Sklar, "Statistical metric spaces," Pacific Journal of Mathematics, vol. 10, pp. 314-334, 1960.

[13] C. Alegre, J. Ferrer, and V. Gregori, "Quasi-uniformities on real vector spaces," The Indian Journal of Pure and Applied Mathematics, vol. 28, pp. 929-937, 1997.

[14] M. Akram, "Fuzzy lie algebras," Infosys Science Foundation Series in Mathematical Sciences, Springer, Berlin, Germany, 2018.

[15] M. Akram, B. Davvaz, and F. Feng, "Fuzzy soft Lie algebras," Journal of Multiple-Valued Logic and Soft Computing, vol. 24, pp. 501-520, 2015.

[16] M. Akram, K. H. Dar, and K. P. Shum, "Interval-valued -fuzzy K-algebras," Applied Soft Computing, vol. 11, no. 1, pp. 1213-1222, 2011.

[17] L. Wang and N. Li, "Pythagorean fuzzy interaction power Bonferroni mean aggregation operators in multiple attribute decision making," International Journal of Intelligent Systems, vol. 35, no. 1, pp. 150-183, 2020. 